Data Article

\title{
Assessing museums' daylighting adequacy without annual measurement campaign: Dataset of a confrontation between measured and simulated illuminance values inside the Cetacean Gallery of the Charterhouse of Calci
}

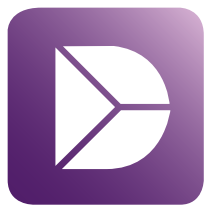

\section{Francesco Leccese ${ }^{a, *}$, Giacomo Salvadori ${ }^{a}{ }^{a}$, Giuseppe Tambellini ${ }^{a}$, Zehra Tugce Kazanasmaz ${ }^{\mathrm{b}}$}

a School of Engineering, University of Pisa, Italy

${ }^{\mathrm{b}}$ Dept. of Architecture, Izmir Institute of Technology, Turkey

\section{A R T I C L E I N F O}

\section{Article history:}

Received 10 July 2020

Accepted 17 July 2020

Available online 24 July 2020

\section{Keywords:}

Daylighting simulations accuracy

Annual measurement campaign

Climate-based daylighting model

Cultural heritage lighting

\begin{abstract}
A B S T R A C T
Lighting Cultural Heritage is a complex task: light is necessary for the act of seeing, it can even enhance the visual experience $[1,2]$, in addition proper lighting can significantly cut down energy consumptions [3], but on the same time it has detrimental effects on exhibits, especially daylight. In order to safeguard the exhibits from damages, national and international standards provide specific recommendations for exhibits' exposure, based on their photosensitivity category. These recommendations are the annual luminous exposure(LO) and the Maximum Illuminance Level ( $\left.E_{\max }\right)$, museums' curators have to verify that the display lighting conditions comply with the standards. Historical buildings are often converted into museums but, as their original purpose was different, the lighting conditions are often inadequate (e.g. too much uncontrolled daylight), therefore the lighting conditions' adequacy of the space should be assessed [4]. As the name suggest the annual luminous exposure requires an annual monitoring campaign, unfortunately it often happens that exhibits have been exposed incorrectly for
\end{abstract}

DOI of original article: 10.1016/j.culher.2020.06.010

* Corresponding authors.

E-mail addresses: f.leccese@ing.unipi.it (F. Leccese), giacomo.salvadori@unipi.it (G. Salvadori). 
prolonged periods, and therefore it is very important to evaluate the need of a fast intervention. In this casuistry a prolonged measurement campaign is not acceptable. Simulations can help running a great number of analysis while reducing the length and expenses of a measurements campaign, however their previsions must be validated. This paper provides the data acquired through measurements and simulations inside the Cetacean Gallery of the Monumental Charterhouse of Calci, near Pisa (Tuscany Region, Italy). The data comprehends horizontal and vertical illuminance measurements, recorded on December the 6th, and simulations run in Grasshopper with the plugins Honeybee+ and Ladybug. The data are related to the research article entitled "Application of climate-based daylight simulation to assess lighting conditions of space and artworks in historical buildings: the case study of Cetacean Gallery of the Monumental Charterhouse of Calci", published on the Journal of Cultural Heritage [5].

(c) 2020 The Author(s). Published by Elsevier Inc.

This is an open access article under the CC BY license.

(http://creativecommons.org/licenses/by/4.0/)

\section{Specifications Table}

Subject
Specific subject area
Type of data
How data were acquired

Data format

Parameters for data collection

Description of data collection

Data source location

Data accessibility

Related research article

\author{
Building Engineering \\ Daylight in buildings \\ Tables, Images and Graphs \\ On-site measurements with luxmeter (Delta OHM 2102.2), simulations run in \\ Grasshopper with Honeybee+ and Ladybug. Simulations rely on climate-based \\ data provided by the Pisa Weather Station. \\ Raw, Analysed \\ Illuminance values were collected in accordance with EN 12,464-1 \\ recommendations [6], for defining measurement grid's dimensions, spacing \\ and minimum number of points. \\ Grasshopper's simulations precision was ensured referring to the recommended \\ Radiance parameters for accurate analyses [7].
}

Illuminance values were measured with Delta Ohm 2102.2 lxmeter, on December the 6th from 10:25 to 12:25. Operators ensured not to cast shadows on the luxmeter sensor using an extension-cable between the sensor and the instrument. Horizontal and vertical illuminances were measured, for the horizontal values a plan placed $1.00 \mathrm{~m}$ above the floor level was used, vertical values were measured twice, first facing south and then north, with the sensor placed $1.50 \mathrm{~m}$ above the floor level. Annual climate-based simulations were run in Grasshopper using the environmental plugins Honeybee+ and Ladybug. Simulations' accuracy was validated though a confrontation with the on-site measurements.

Natural History Museum of the University of Pisa, housed inside the Monumental Charterhouse of Calci, Pisa (Tuscany Region, Italy), geographic coordinates: $43^{\circ} 43^{\prime} 19^{\prime \prime} \mathrm{N}, 10^{\circ} 31^{\prime} 22^{\prime \prime} \mathrm{E}$.

Data are within this article; climate-based data ("epw" file format or others) can be free downloaded at https://energyplus.net/weather-location/ europe_wmo_region_6/ITA//ITA_Pisa.161580_IWEC.

"Application of climate-based daylight simulation to assess lighting conditions of space and artworks in historical buildings: the case study of Cetacean Gallery of the Monumental Charterhouse of Calci", Authors: F. Leccese, G. Salvadori, G. Tambellini, Z.T. Kazanasmaz, Journal of Cultural Heritage [5].

\section{Value of the Data}

- The data in this article demonstrates that lighting software simulations can substitute prolonged measurement campaign, if the 3D model is well calibrated. 
- Lighting designers can use these data as reference for comparing illuminance on-site measurements and simulations' accuracy in similar contexts.

- The data can be used as a basis to further inquire about the daylighting adequacy inside the Natural History Museum.

\section{Data}

The data shown in this article are related to the research paper entitled "Application of climate-based daylight simulation to assess lighting conditions of space and artworks in historical buildings: the case study of Cetacean Gallery of the Monumental Charterhouse of Calci" [5]. The data validate simulations' previsions with additional confrontations between the measured illuminance values and the software simulated ones; the data are referred to the Cetacean Gallery of the Charterhouse of Calci. The Gallery has a rectangular plan $(110 \times 7 \mathrm{~m})$ divided in 21 bays by brick columns. Three of the four vertical surfaces are almost entirely windowed, leading to a ratio of window to floor area of $67 \%$. With its net volume of $3426 \mathrm{~m}^{3}$ and floor area of 699 $\mathrm{m}^{2}$, the Gallery is the largest exhibition room of the Natural History Museum, housed inside the Monumental Charterhouse of Calci, near Pisa. The space corresponds with the monastery's ex-barn and hosts the most important cetacean skeleton collection in Italy, composed by 28 skeletons, 8 fossils, 47 life-sized and scale models, and 9 thematic areas.

Table 1 provides four recommended Radiance parameter settings. The settings vary depending on the level of accuracy required in the simulations. The last row of Table 1 provides a comparison with the parameter settings used in the research. Fig. 1 is a visual representation of the grids used during the measurement campaign. Floor plan and sections are displayed. Measurements points are highlighted in blue, non-accessible area (due to the exhibits' presence) are highlighted in cyan. Table 2, Table 3 and Table 4 provide the comparison between the illuminance values that were measured on-site and those that were obtained through the simulations. Simulations results comprehends four columns of values, depending on how the results were obtained: PIT values were obtained through point-in-time analysis using Climate-Based sky (CB), CIE Clear sky and CIE Overcast sky respectively. Finally results read from annual analysis with Climate-based sky (RFA) are displayed. Fig. 2, Fig. 3 and Fig. 4 are visual representations of the previous tables. The measurements points are placed on the x-axis, the illuminance levels are placed on the y-axis. Results are differentiated with symbols.

\section{Experimental Design, Materials, and Methods}

Illuminance levels were measured inside the Cetacean Gallery of the Charterhouse of Calci using Delta Ohm 2102.2 lxmeter on December the 6th from 10:25 to 12:25. The measurement grid was defined according to EN 12,464-1 recommendations [6] based on the room's geometry: as the Gallery measures $110 \times 7 \mathrm{~m}$ the standard sets the minimum number of points to 20 and their spacing to $5.0 \mathrm{~m}$. The grid used for the on-site measurements is composed by 58 points, spaced $3.00 \times 1.75 \mathrm{~m}$ (Fig. 1). Horizontal Illuminance was measured on a horizonal plane

Table 1

Recommended Radiance parameters [7], depending on the required analysis accuracy.

\begin{tabular}{lllll}
\hline Required analysis accuracy & ab & aa & ar & ad \\
\hline Minimum & 0 & 0.5 & 8 & 0 \\
Fast & 0 & 0.2 & 32 & 32 \\
Accurate & 2 & 0.15 & 128 & 512 \\
Maximum & 8 & 0.0 & 0 & 4096 \\
Value used in simulations & 2 & 0.1 & 300 & 1000 \\
\hline
\end{tabular}


Table 2

Horizontal illuminance values (lx); sensor placed $1.00 \mathrm{~m}$ above floor level.

\begin{tabular}{|c|c|c|c|c|c|c|}
\hline $\begin{array}{l}\text { Grid point } \\
\text { ID }\end{array}$ & Hour & $\begin{array}{l}\text { On-site } \\
\text { measurements }\end{array}$ & $\begin{array}{l}\text { Simulations result } \\
\text { PIT Climate-based } \\
\text { sky }\end{array}$ & $\begin{array}{l}\text { RFA Climate-based } \\
\text { sky }\end{array}$ & $\begin{array}{l}\text { PIT CIE Clear } \\
\text { sky }\end{array}$ & $\begin{array}{l}\text { PIT CIE } \\
\text { Overcast sky }\end{array}$ \\
\hline $\mathbf{0}$ & $12: 25$ & 82 & 2770 & 5119 & 1405 & 621 \\
\hline 1 & $12: 24$ & 226 & 18,833 & 20,953 & 22,800 & 998 \\
\hline 2 & $12: 23$ & 393 & 1384 & 1931 & 877 & 1001 \\
\hline 3 & $12: 23$ & 408 & 1201 & 1387 & 642 & 966 \\
\hline 4 & $12: 22$ & 463 & 886 & 1260 & 493 & 994 \\
\hline 5 & $12: 22$ & 352 & 21,823 & 19,059 & 23,927 & 828 \\
\hline 6 & $12: 25$ & 126 & 1876 & 2264 & 1038 & 812 \\
\hline 7 & $12: 24$ & 217 & 22,319 & 25,821 & 24,183 & 495 \\
\hline 8 & $12: 23$ & 280 & 2028 & 2456 & 1161 & 489 \\
\hline 9 & $12: 22$ & 268 & 21,680 & 21,972 & 23,919 & 474 \\
\hline 10 & $12: 25$ & 260 & 21,461 & 24,078 & 23,869 & 756 \\
\hline 11 & $12: 24$ & 144 & 22,741 & 24,587 & 24,378 & 455 \\
\hline 12 & $12: 22$ & 332 & 20,156 & 22,964 & 23,332 & 502 \\
\hline 13 & $12: 25$ & 133 & 20,343 & 20,096 & 23,370 & 144 \\
\hline 14 & $12: 21$ & 381 & 21,185 & 20,417 & 23,652 & 710 \\
\hline 15 & $12: 21$ & 488 & 2009 & 3134 & 1261 & 851 \\
\hline 16 & $12: 21$ & 450 & 2826 & 7260 & 1425 & 666 \\
\hline 17 & $12: 20$ & 185 & 2026 & 2452 & 1078 & 810 \\
\hline 18 & $12: 20$ & 426 & 2493 & 2948 & 1360 & 725 \\
\hline 19 & $12: 20$ & 471 & 22,277 & 22,603 & 24,050 & 762 \\
\hline 20 & $12: 20$ & 443 & 23,253 & 23,951 & 24,471 & 777 \\
\hline 21 & $12: 19$ & 413 & 1713 & 1955 & 771 & 708 \\
\hline 22 & $12: 19$ & 462 & 2747 & 2946 & 1258 & 821 \\
\hline 23 & $12: 19$ & 446 & 2825 & 3083 & 1416 & 665 \\
\hline 24 & $12: 18$ & 482 & 2953 & 3815 & 1566 & 790 \\
\hline 25 & $12: 18$ & 437 & 3098 & 3427 & 1564 & 715 \\
\hline 26 & $12: 18$ & 432 & 2850 & 3590 & 1443 & 746 \\
\hline 27 & $12: 18$ & 527 & 2515 & 2828 & 1161 & 748 \\
\hline 28 & $12: 17$ & 421 & 19,686 & 20,255 & 23,193 & 630 \\
\hline 29 & $12: 17$ & 503 & 19,916 & 20,681 & 23,194 & 766 \\
\hline 30 & $12: 16$ & 422 & 20,126 & 20,910 & 23,376 & 651 \\
\hline 31 & $12: 16$ & 438 & 1934 & 2057 & 993 & 745 \\
\hline 32 & $12: 16$ & 432 & 21,056 & 20,740 & 23,553 & 695 \\
\hline 33 & $12: 16$ & 340 & 21,333 & 23,222 & 23,804 & 724 \\
\hline 34 & $12: 15$ & 427 & 630 & 770 & 419 & 754 \\
\hline 35 & $12: 15$ & 363 & 22,628 & 21,843 & 24,269 & 649 \\
\hline 36 & $12: 14$ & 423 & 23,376 & 21,347 & 24,625 & 787 \\
\hline 37 & $12: 14$ & 366 & 4689 & 5965 & 2145 & 696 \\
\hline 38 & $12: 14$ & 380 & 23,418 & 21,065 & 24,579 & 746 \\
\hline 39 & $12: 09$ & 186 & 22,136 & 23,095 & 24,106 & 246 \\
\hline 40 & $12: 09$ & 50 & 23,241 & 21,866 & 24,486 & 134 \\
\hline 41 & $12: 08$ & 52 & 23,210 & 22,684 & 24,451 & 131 \\
\hline 42 & $12: 08$ & 77 & 22,644 & 22,518 & 24,172 & 198 \\
\hline 43 & $12: 08$ & 128 & 23,314 & 23,219 & 24,529 & 206 \\
\hline 44 & $12: 12$ & 159 & 4143 & 6990 & 2052 & 478 \\
\hline 45 & 12:09 & 166 & 23,224 & 24,535 & 24,542 & 637 \\
\hline 46 & $12: 12$ & 332 & 22,402 & 18,890 & 24,186 & 559 \\
\hline 47 & $12: 10$ & 211 & 23,068 & 23,271 & 24,467 & 760 \\
\hline 48 & $12: 13$ & 349 & 23,044 & 21,872 & 24,441 & 456 \\
\hline 49 & $12: 13$ & 186 & 22,367 & 25,171 & 24,086 & 556 \\
\hline 50 & $12: 13$ & 438 & 23,266 & 23,155 & 24,462 & 688 \\
\hline 51 & $12: 11$ & 173 & 4235 & 4262 & 2061 & 323 \\
\hline 52 & $12: 11$ & 169 & 23,187 & 24,499 & 24,487 & 339 \\
\hline 53 & $12: 11$ & 327 & 22,603 & 21,418 & 24,303 & 413 \\
\hline 54 & $12: 10$ & 110 & 22,755 & 24,259 & 24,318 & 300 \\
\hline
\end{tabular}


Table 2 (continued)

\begin{tabular}{|c|c|c|c|c|c|c|}
\hline $\begin{array}{l}\text { Grid point } \\
\text { ID }\end{array}$ & Hour & $\begin{array}{l}\text { On-site } \\
\text { measurements }\end{array}$ & $\begin{array}{l}\text { Simulations results } \\
\text { PIT Climate-based } \\
\text { sky }\end{array}$ & $\begin{array}{l}\text { RFA Climate-based } \\
\text { sky }\end{array}$ & $\begin{array}{l}\text { PIT CIE Clear } \\
\text { sky }\end{array}$ & $\begin{array}{l}\text { PIT CIE } \\
\text { Overcast sky }\end{array}$ \\
\hline 55 & $12: 11$ & 184 & 23,161 & 21,805 & 24,493 & 387 \\
\hline 56 & $12: 10$ & 217 & 21,691 & 21,370 & 23,940 & 624 \\
\hline 57 & $12: 11$ & 246 & 23,132 & 22,779 & 24,503 & 929 \\
\hline \multicolumn{2}{|c|}{$\begin{array}{l}\text { Average illuminance } \\
{[\mathbf{l x}]}\end{array}$} & 310 & 14,653 & 15,084 & 15,416 & 625 \\
\hline \multicolumn{2}{|l|}{ MD [\%] } & 1 & +46.2 & +47.6 & +48.7 & +1.01 \\
\hline
\end{tabular}

placed $1.00 \mathrm{~m}$ above floor level, vertical illuminance was measured $1.50 \mathrm{~m}$ above floor level both in north and south direction. The measured values (Tables 2-4) were then confronted with software simulations (Figs. 2-4) in order to verify their accuracy. Simulations' grid was denser, with a total of 287 measurements points spaced $1.50 \times 1.75 \mathrm{~m}$. However, to maximize the confrontation's significance, some points were excluded and just the exact same ones of the on-site grid were used for the confrontation. Simulations were run in Grasshopper using Honeybee+ and Ladybug, Radiance-based environmental plugins (Table 1). The 3D model of the Gallery is based on an architectural survey conducted by the University of Pisa, the geometries were modelled in Rhinoceros and then imported in Grasshopper. Two kinds of simulation were run: point-intime (PIT) and annual. PIT simulations are implemented in every lighting software, Annual ones are not. Annual analyses are more accurate, on account of using climate-based data for the sky model creation: the climate-based data provides the TMY (typical meteorological year) for the examined site. The TMY is composed by the succession of the most recurrent weather conditions observed during the recording period [8]. For the data used in simulations the recording
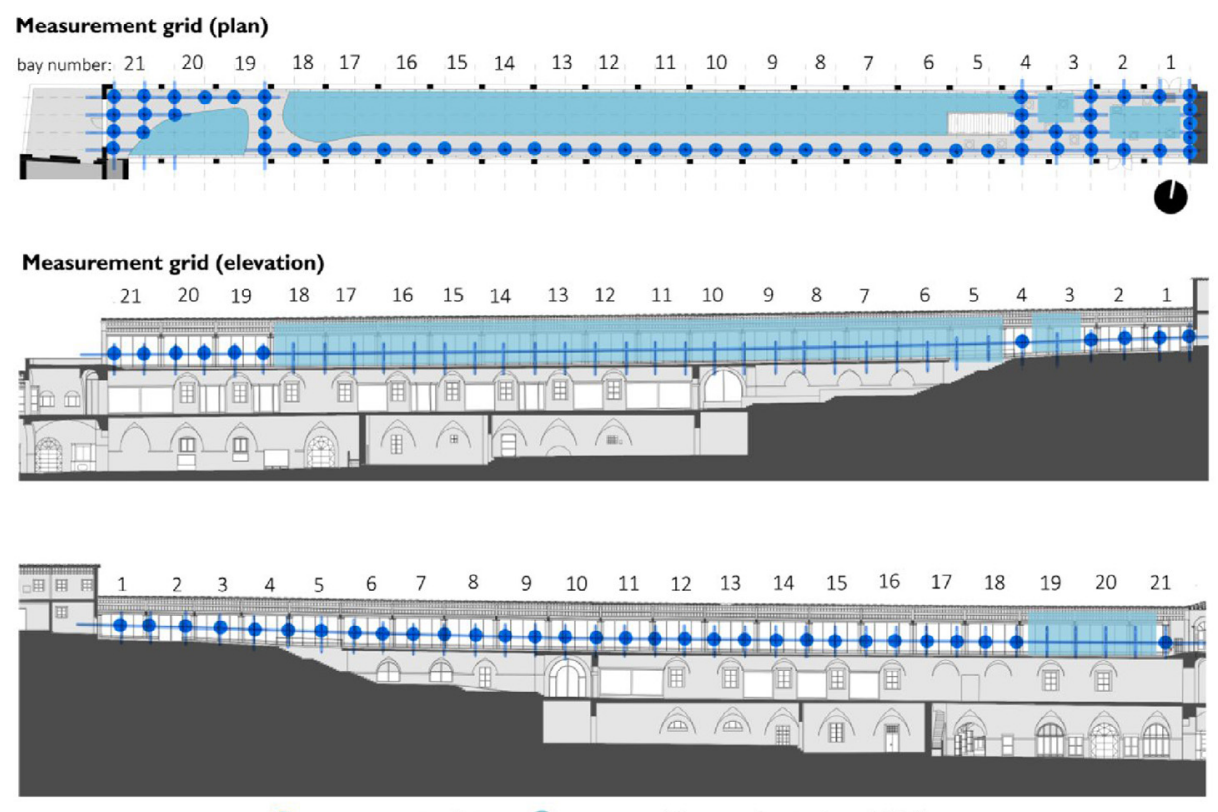

measurement points

non-accessible areas due to the exhibits' presence

Fig. 1. Cetacean Gallery: measurement grid (plan and elevations). 
Table 3

Vertical illuminance values (lx); sensor facing south $1.50 \mathrm{~m}$ above floor level.

\begin{tabular}{|c|c|c|c|c|c|c|}
\hline $\begin{array}{l}\text { Grid point } \\
\text { ID }\end{array}$ & Hour & $\begin{array}{l}\text { On-site } \\
\text { measurements }\end{array}$ & $\begin{array}{l}\text { Simulations result } \\
\text { PIT Climate-based } \\
\text { sky }\end{array}$ & $\begin{array}{l}\text { RFA Climate-based } \\
\text { sky }\end{array}$ & $\begin{array}{l}\text { PIT CIE Clear } \\
\text { sky }\end{array}$ & $\begin{array}{l}\text { PIT CIE } \\
\text { Overcast sky }\end{array}$ \\
\hline 0 & $10: 23$ & 146 & 6690 & 10,613 & 3432 & 583 \\
\hline 1 & $10: 24$ & 207 & 5467 & 7277 & 3122 & 403 \\
\hline 2 & $10: 25$ & 188 & 4324 & 5374 & 2796 & 273 \\
\hline 3 & $10: 26$ & 213 & 3494 & 4402 & 2585 & 200 \\
\hline 4 & $10: 26$ & 304 & 3021 & 4003 & 2102 & 191 \\
\hline 5 & $10: 26$ & 378 & 51,589 & 55,561 & 55,387 & 1198 \\
\hline 6 & $10: 23$ & 207 & 4774 & 5062 & 3512 & 303 \\
\hline 7 & $10: 24$ & 306 & 53,412 & 55,578 & 56,565 & 1333 \\
\hline 8 & $10: 25$ & 186 & 5592 & 5837 & 4063 & 370 \\
\hline 9 & $10: 27$ & 572 & 50,682 & 50,418 & 54,980 & 1126 \\
\hline 10 & $10: 23$ & 293 & 50,070 & 49,093 & 54,614 & 1200 \\
\hline 11 & $10: 24$ & 270 & 53,702 & 52,120 & 56,207 & 1501 \\
\hline 12 & $10: 28$ & 1041 & 9944 & 5921 & 5393 & 810 \\
\hline 13 & $10: 23$ & 49 & 9889 & 9553 & 5253 & 861 \\
\hline 14 & $10: 28$ & 1412 & 49,932 & 48,696 & 54,935 & 950 \\
\hline 15 & $10: 47$ & 2253 & 7370 & 8233 & 4729 & 529 \\
\hline 16 & $10: 47$ & 1718 & 8295 & 8840 & 4978 & 597 \\
\hline 17 & $10: 48$ & 2289 & 5690 & 6515 & 4090 & 381 \\
\hline 18 & $10: 48$ & 2117 & 6543 & 7368 & 4489 & 450 \\
\hline 19 & $10: 48$ & 2195 & 51,112 & 46,082 & 55,087 & 1440 \\
\hline 20 & $10: 49$ & 2406 & 53,992 & 55,703 & 56,299 & 1535 \\
\hline 21 & $10: 51$ & 1606 & 2052 & 1868 & 1211 & 308 \\
\hline 22 & $10: 51$ & 2137 & 5352 & 5634 & 3442 & 403 \\
\hline 23 & $10: 52$ & 1714 & 6304 & 6547 & 4111 & 437 \\
\hline 24 & $10: 52$ & 1985 & 6751 & 6941 & 4495 & 478 \\
\hline 25 & $10: 52$ & 1928 & 6766 & 7084 & 4409 & 452 \\
\hline 26 & $10: 53$ & 1772 & 6977 & 7390 & 4506 & 475 \\
\hline 27 & $10: 53$ & 1983 & 3031 & 3229 & 1522 & 595 \\
\hline 28 & $10: 53$ & 1530 & 7321 & 9544 & 4086 & 527 \\
\hline 29 & $10: 53$ & 1961 & 8374 & 8718 & 4666 & 602 \\
\hline 30 & $10: 54$ & 1655 & 8786 & 9968 & 4974 & 653 \\
\hline 31 & $11: 34$ & 1858 & 1629 & 1591 & 921 & 306 \\
\hline 32 & $11: 35$ & 1989 & 48,169 & 44,638 & 53,838 & 794 \\
\hline 33 & $11: 35$ & 1573 & 49,285 & 47,050 & 54,070 & 948 \\
\hline 34 & $11: 35$ & 1971 & 551 & 566 & 375 & 103 \\
\hline 35 & $11: 36$ & 1525 & 50,881 & 52,875 & 54,592 & 1260 \\
\hline 36 & $11: 36$ & 1940 & 53,174 & 51,928 & 55,532 & 1559 \\
\hline 37 & $11: 36$ & 1415 & 10,228 & 10,298 & 4780 & 1228 \\
\hline 38 & $11: 36$ & 1811 & 53,273 & 52,044 & 55,536 & 1523 \\
\hline 39 & $11: 39$ & 467 & 49,627 & 51,386 & 54,101 & 1321 \\
\hline 40 & $11: 40$ & 235 & 52,998 & 53,999 & 55,284 & 1462 \\
\hline 41 & $11: 40$ & 161 & 52,866 & 52,464 & 55,095 & 1462 \\
\hline 42 & $11: 40$ & 119 & 51,012 & 51,325 & 54,573 & 1283 \\
\hline 43 & $11: 40$ & 110 & 53,509 & 52,391 & 55,590 & 1552 \\
\hline 44 & $11: 39$ & 581 & 9224 & 3664 & 4219 & 1215 \\
\hline 45 & $11: 41$ & 162 & 53,285 & 54,623 & 55,662 & 1543 \\
\hline 46 & $11: 39$ & 942 & 51,082 & 51,182 & 54,772 & 1359 \\
\hline 47 & $11: 41$ & 203 & 52,774 & 54,094 & 55,294 & 1417 \\
\hline 48 & $11: 38$ & 1127 & 53,248 & 53,128 & 55,612 & 1527 \\
\hline 49 & $11: 38$ & 337 & 50,368 & 55,788 & 54,456 & 1219 \\
\hline 50 & $11: 38$ & 1527 & 53,512 & 56,063 & 55,870 & 1562 \\
\hline 51 & $11: 42$ & 484 & 9885 & 10,459 & 4884 & 1261 \\
\hline 52 & $11: 43$ & 411 & 53,441 & 56,871 & 55,913 & 1513 \\
\hline 53 & $11: 43$ & 673 & 52,404 & 52,554 & 55,268 & 1406 \\
\hline 54 & $11: 42$ & 286 & 52,467 & 52,383 & 55,566 & 1358 \\
\hline
\end{tabular}


Table 3 (continued)

\begin{tabular}{|c|c|c|c|c|c|c|}
\hline $\begin{array}{l}\text { Grid point } \\
\text { ID }\end{array}$ & Hour & $\begin{array}{l}\text { On-site } \\
\text { measurements }\end{array}$ & $\begin{array}{l}\text { Simulations results } \\
\text { PIT Climate-based } \\
\text { sky }\end{array}$ & $\begin{array}{l}\text { RFA Climate-based } \\
\text { sky }\end{array}$ & $\begin{array}{l}\text { PIT CIE Clear } \\
\text { sky }\end{array}$ & $\begin{array}{l}\text { PIT CIE } \\
\text { Overcast sky }\end{array}$ \\
\hline 55 & $11: 43$ & 427 & 53,591 & 52,994 & 55,967 & 1550 \\
\hline 56 & $11: 42$ & 225 & 49,300 & 52,176 & 54,270 & 1240 \\
\hline 57 & $11: 44$ & 284 & 53,995 & 57,583 & 56,186 & 1567 \\
\hline \multicolumn{2}{|c|}{$\begin{array}{l}\text { Average illuminance } \\
{[\mathbf{l x}]}\end{array}$} & 1032 & 29,881 & 30,264 & 30,349 & 960 \\
\hline \multicolumn{2}{|l|}{ MD [\%] } & 1 & +28.0 & +28.3 & +28.4 & +0.07 \\
\hline
\end{tabular}

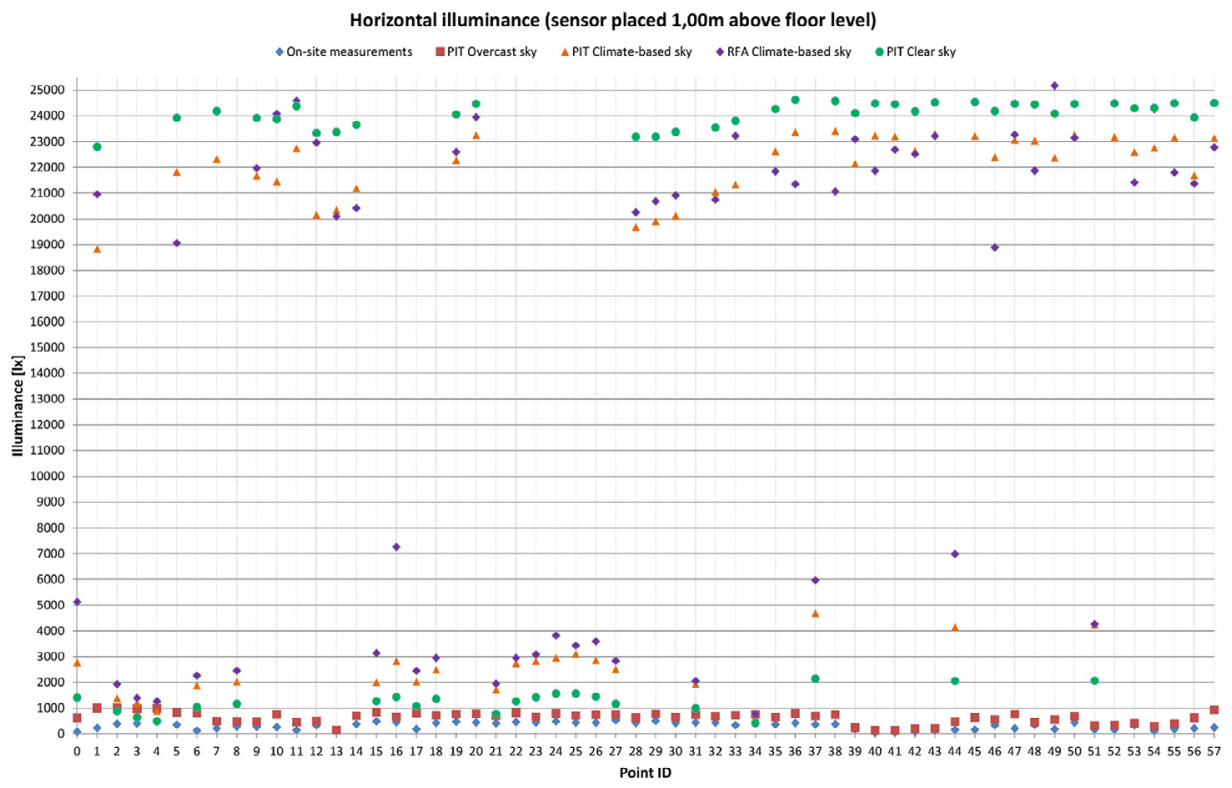

Fig. 2. Horizontal Illuminance values on the selected points' grid.

period is $1982-1997$. The mean deviation (MD) is calculated as (Tables 2-4):

$$
M D=\frac{\sum_{i=1}^{n} E_{S, i}-\sum_{i=1}^{n} E_{M, i}}{\sum_{i=1}^{n} E_{M, i}} \%
$$

where: $\mathrm{i}(1,2, \ldots, \mathrm{n})$ are the grid points, $\mathrm{E}_{S, \mathrm{i}}$ the illuminance values obtained through the simulations, $\mathrm{E}_{\mathrm{M}, \mathrm{i}}$ the illuminance values obtained through the on-site measurements.

\section{Funding}

This research was partially funded by the University of Pisa as part of the biennial project: 〈Technical committee for the predisposition of cognitive studies aimed to the restoration, the conservation and the enhancement of the Charterhouse of Calci and its Museums (2017-2019), University of Pisa board resolution $\mathrm{N}^{\circ} 7 / 2017$, concerning thermal, acoustic and lighting analysis. The project involves the University of Pisa (Technical Office for the Management and the Maintenance Activities on the building heritage, and School of Engineering), the Italian Ministry of Cultural Heritage and Activities, the Italian Heritage Protection Department. 
Table 4

Vertical illuminance values (lx); sensor facing north $1.50 \mathrm{~m}$ above floor level.

\begin{tabular}{|c|c|c|c|c|c|c|}
\hline $\begin{array}{l}\text { Grid point } \\
\text { ID }\end{array}$ & Hour & $\begin{array}{l}\text { On-site } \\
\text { measurements }\end{array}$ & $\begin{array}{l}\text { Simulations result } \\
\text { PIT Climate-based } \\
\text { sky }\end{array}$ & $\begin{array}{l}\text { sFA Climate-based } \\
\text { sky }\end{array}$ & $\begin{array}{l}\text { PIT CIE Clear } \\
\text { sky }\end{array}$ & $\begin{array}{l}\text { PIT CIE } \\
\text { Overcast sky }\end{array}$ \\
\hline 0 & 1 & 1 & 2193 & 2247 & 1980 & 214 \\
\hline 1 & $10: 31$ & 416 & 2160 & 2667 & 1857 & 255 \\
\hline 2 & $10: 31$ & 950 & 1912 & 2256 & 1451 & 342 \\
\hline 3 & $10: 31$ & 931 & 1778 & 2090 & 1034 & 473 \\
\hline 4 & $10: 30$ & 990 & 1497 & 1880 & 817 & 425 \\
\hline 5 & $10: 30$ & 870 & 3102 & 3336 & 2873 & 336 \\
\hline 6 & $10: 32$ & 87 & 4272 & 4722 & 2293 & 1540 \\
\hline 7 & 1 & 1 & 3558 & 3604 & 3192 & 407 \\
\hline 8 & 1 & 1 & 4658 & 5565 & 2523 & 1621 \\
\hline 9 & $10: 29$ & 561 & 3632 & 4451 & 3308 & 411 \\
\hline 10 & $10: 32$ & 114 & 3676 & 4175 & 3211 & 467 \\
\hline 11 & 1 & 1 & 4167 & 4426 & 3620 & 500 \\
\hline 12 & $10: 29$ & 389 & 3673 & 4268 & 3038 & 537 \\
\hline 13 & $10: 33$ & 204 & 3923 & 4177 & 3126 & 597 \\
\hline 14 & $10: 29$ & 303 & 4278 & 4692 & 3510 & 631 \\
\hline 15 & $10: 58$ & 412 & 3686 & 4271 & 2571 & 850 \\
\hline 16 & $10: 58$ & 337 & 4380 & 4940 & 3120 & 999 \\
\hline 17 & $10: 58$ & 286 & 3986 & 4688 & 2193 & 1366 \\
\hline 18 & $10: 57$ & 294 & 5070 & 5785 & 2736 & 1847 \\
\hline 19 & $10: 57$ & 337 & 3937 & 4123 & 3323 & 532 \\
\hline 20 & $10: 57$ & 355 & 3852 & 4036 & 3295 & 487 \\
\hline 21 & $10: 57$ & 358 & 3280 & 3553 & 1858 & 1217 \\
\hline 22 & $10: 56$ & 311 & 5605 & 6603 & 3384 & 1909 \\
\hline 23 & $10: 56$ & 295 & 5370 & 5868 & 3150 & 1845 \\
\hline 24 & $10: 56$ & 278 & 5302 & 6307 & 3237 & 1796 \\
\hline 25 & $10: 56$ & 293 & 5643 & 6572 & 3328 & 1939 \\
\hline 26 & $10: 55$ & 274 & 4714 & 5647 & 2847 & 1533 \\
\hline 27 & $10: 55$ & 271 & 2723 & 3391 & 1738 & 751 \\
\hline 28 & $10: 54$ & 294 & 4547 & 4578 & 3294 & 1048 \\
\hline 29 & $10: 54$ & 283 & 4830 & 5064 & 3502 & 1051 \\
\hline 30 & $10: 54$ & 276 & 4779 & 5708 & 3605 & 1019 \\
\hline 31 & $11: 51$ & 180 & 3315 & 3643 & 2073 & 912 \\
\hline 32 & $11: 51$ & 173 & 4097 & 4543 & 3207 & 670 \\
\hline 33 & $11: 50$ & 182 & 4599 & 5438 & 3684 & 717 \\
\hline 34 & $11: 50$ & 184 & 2092 & 2154 & 1474 & 425 \\
\hline 35 & $11: 50$ & 182 & 4435 & 5315 & 3666 & 565 \\
\hline 36 & $11: 49$ & 152 & 4513 & 5178 & 3862 & 596 \\
\hline 37 & $11: 49$ & 132 & 4359 & 5105 & 3617 & 572 \\
\hline 38 & $11: 49$ & 97 & 4494 & 4628 & 3889 & 598 \\
\hline 39 & $11: 47$ & 69 & 4333 & 4846 & 3576 & 616 \\
\hline 40 & $11: 47$ & 83 & 4470 & 4847 & 3829 & 570 \\
\hline 41 & $11: 47$ & 138 & 4359 & 5213 & 3755 & 588 \\
\hline 42 & $11: 47$ & 292 & 4376 & 4799 & 3732 & 601 \\
\hline 43 & $11: 46$ & 428 & 4479 & 5098 & 3780 & 583 \\
\hline 44 & $11: 48$ & 99 & 4413 & 4996 & 3633 & 575 \\
\hline 45 & $11: 46$ & 434 & 4581 & 4588 & 3832 & 589 \\
\hline 46 & $11: 48$ & 129 & 4359 & 4931 & 3622 & 563 \\
\hline 47 & $11: 46$ & 596 & 4409 & 5036 & 3808 & 592 \\
\hline 48 & $11: 48$ & 109 & 4377 & 5339 & 3761 & 575 \\
\hline 49 & $11: 48$ & 108 & 4357 & 5208 & 3663 & 596 \\
\hline 50 & $11: 48$ & 114 & 4457 & 5079 & 3834 & 560 \\
\hline 51 & $11: 45$ & 132 & 4255 & 4681 & 3592 & 568 \\
\hline 52 & $11: 45$ & 128 & 4451 & 4489 & 3788 & 609 \\
\hline 53 & $11: 45$ & 161 & 4218 & 4465 & 3602 & 565 \\
\hline 54 & $11: 45$ & 252 & 4347 & 4646 & 3704 & 563 \\
\hline
\end{tabular}


Table 4 (continued)

\begin{tabular}{|c|c|c|c|c|c|c|}
\hline $\begin{array}{l}\text { Grid point } \\
\text { ID }\end{array}$ & Hour & $\begin{array}{l}\text { On-site } \\
\text { measurements }\end{array}$ & $\begin{array}{l}\text { Simulations results } \\
\text { PIT Climate-based } \\
\text { sky }\end{array}$ & $\begin{array}{l}\text { RFA Climate-based } \\
\text { sky }\end{array}$ & $\begin{array}{l}\text { PIT CIE Clear } \\
\text { sky }\end{array}$ & $\begin{array}{l}\text { PIT CIE } \\
\text { Overcast sky }\end{array}$ \\
\hline 55 & $11: 44$ & 313 & 4349 & 4575 & 3702 & 534 \\
\hline 56 & $11: 46$ & 630 & 4199 & 5102 & 3591 & 546 \\
\hline 57 & $11: 44$ & 708 & 4312 & 4627 & 3781 & 569 \\
\hline \multicolumn{2}{|c|}{$\begin{array}{l}\text { Average illuminance } \\
\text { [lx] }\end{array}$} & 293 & 4055 & 4556 & 3122 & 775 \\
\hline \multicolumn{2}{|l|}{ MD [\%] } & 1 & +12.9 & +14.6 & +9.67 & +1.65 \\
\hline
\end{tabular}

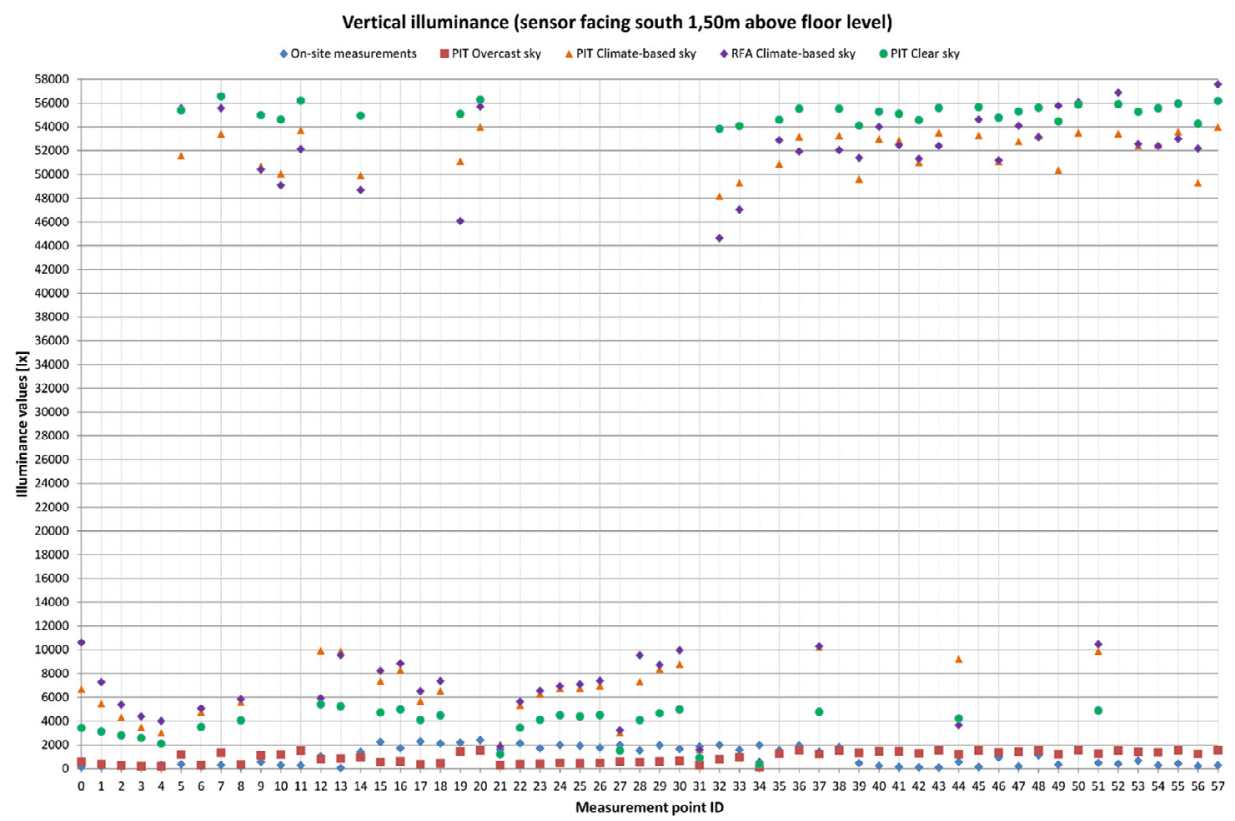

Fig. 3. Vertical illuminance values (facing south) on the selected points' grid.

\section{List of abbreviations}

$\mathrm{ab}$

aа

ar

ad

$\mathrm{E}_{\mathrm{S}, \mathrm{i}}$

$\mathrm{E}_{\mathrm{M}, \mathrm{i}}$

MD

PIT

RFA ambient bounces, it sets the number of diffuse bounces computed in the indirect illuminance calculation

ambient accuracy, it influences the error from indirect illuminance interpolation ambient resolution, it sets the density of the ambient for the interpolation ambient divisions, it influences Monte Carlo's error during indirect illuminance calculation

illuminance values obtained through the simulations illuminance values obtained through the on-site measurements mean deviation, deviation between simulated and measure illuminance values Point In Time, illuminance values obtained through point in time analyses simulations Read From Annual, Illuminance values obtained through annual, climate-based simulations 


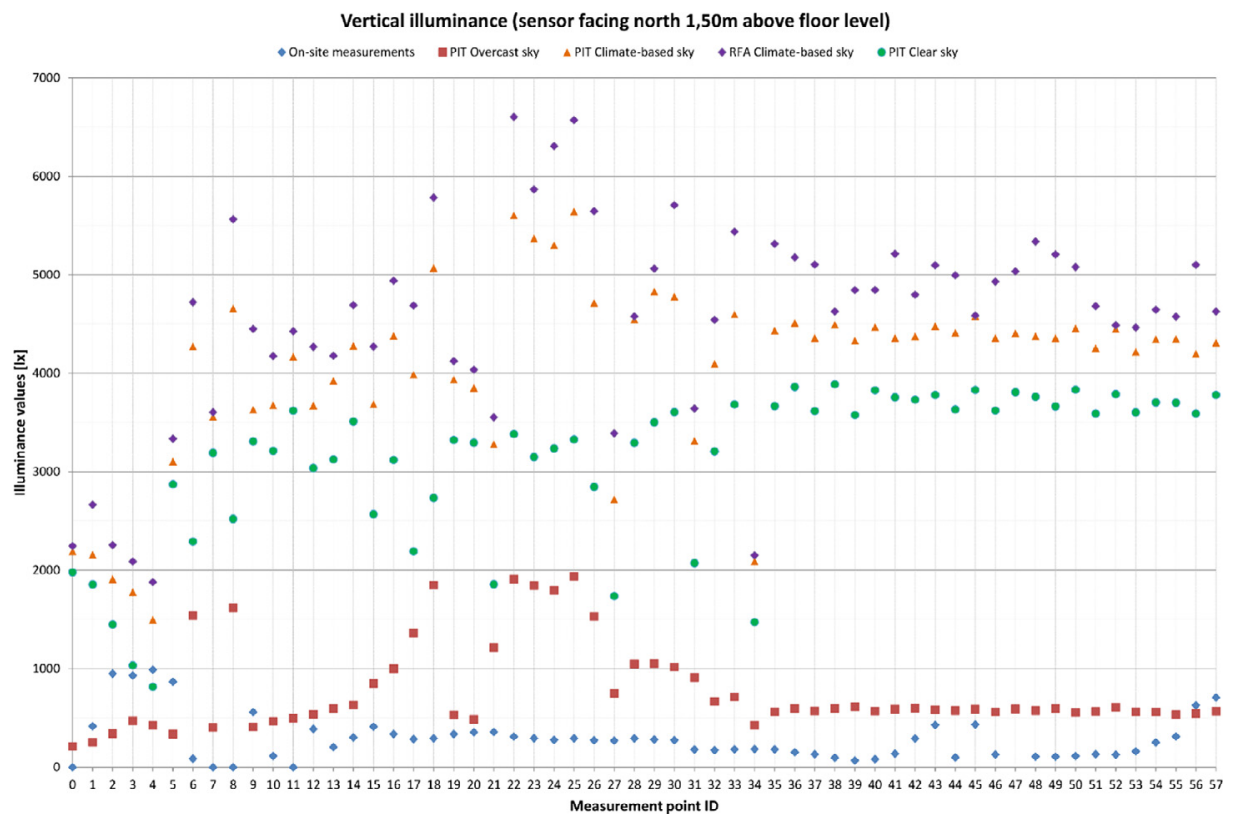

Fig. 4. Vertical illuminance values (facing north) on the selected points' grid.

\section{Declaration of Competing Interest}

The authors declare that they have no known competing financial interests or personal relationships which could have influenced the work reported in this article.

\section{References}

[1] F. Leccese, G. Salvadori, D. Maccheroni, F. Feltrin, Lighting and visual experience of artworks: results of a study campaign at the National Museum of San Matteo in Pisa (Italy), J. Cult. Herit. (2020) 1-11 in press https://doi.org/ 10.1016/j.culher.2020.03.007 .

[2] F. Leccese, G. Salvadori, F. Feltrin, R. Morozzi, P. Nieri, Study on the suitable lighting design of Beato Angelico's artworks displayed at the National Museum of San Matteo in Pisa (Italy), IOP Conf. Ser. Mater. Sci. Eng. 364 (2018) 1-8 https://doi.org/10.1088/1757-899X/364/1/012095.

[3] F. Fantozzi, F. Leccese, G. Salvadori, M. Rocca, I. Capranelli, Opportunities for energy savings with interventions on the lighting systems of historical buildings: the case of "Palazzo Medici" in Pisa (Italy), in: 16th International Conference on Environment and Electrical Engineering (EEEIC), Florence (I), 7-10 June, 2016, pp. 1-6. https://doi.org/10.1109/ EEEIC.2016.7555569.

[4] C. Balocco, M. Cecchi, G. Volante, Natural lighting for sustainability of cultural heritage refurbishment, Sustainability 11 (2019) 1-17 https://doi.org/10.3390/su11184842.

[5] F. Leccese, G. Salvadori, G. Tambellini, Z.T. Kazanasmaz, Application of climate-based daylight simulation to assess lighting conditions of space and artworks in historical buildings: the case study of Cetacean gallery of the monumental charterhouse of Calci, J. Cult. Herit. (2020) 1-14 in press https://doi.org/10.1016/j.culher.2020.06.010 .

[6] EN 12464, Light and lighting-Lighting of work places-Part 1: Indoor work places, Bruxelles (B) 2011.

[7] Website of Radiance, recommended Radiance rendering options (<https://floyd.lbl.gov/radiance/refer/Notes/rpict_ options.html> last accessed 8th May 2020).

[8] Website of the Perez sky model (https://docs.agi32.com/AGi32/Content/references/Perez_All-Weather_Sky_Model.htm last accessed 8th May 2020). 